\title{
Perfil clínico e epidemiológico de crianças com toxoplasmose congênita em instituto de perinatologia de referência
}

\author{
Clinical and epidemiological profile of children with congenital toxoplasmosis in a reference \\ institute of perinatology \\ Perfil clínico y epidemiológico de niños con toxoplasmosis congénita en un instituto de perinatología \\ de referencia
}

Recebido: 08/12/2021 | Revisado: 12/12/2021 | Aceito: 13/12/2021 | Publicado: 21/12/2021

Conceição de Maria Monteiro Benvindo Falcão
ORCID: https://orcid.org/0000-0001-6523-3663
Centro Universitário UNINOVAFAPI, Brasil
E-mail: concabenvindo@gmail.com
Andressa Mikaely Alves de Sousa
ORCID: https://orcid.org/0000-0002-1784-6307
Centro Universitário UNINOVAFAPI, Brasil
E-mail: andressa.mikaely@ hotmail.com
Wiltamara Lacerda de Moura
ORCID: https://orcid.org/0000-0002-8048-0977
Centro Universitário UNINOVAFAPI, Brasil
E-mail: wiltamaralacerda@ gmail.com
Luiza Ivete Vieira Batista
ORCID: https://orcid.org/0000-0003-0208-1062
Centro Universitário UNINOVAFAPI, Brasil
E-mail: luizaivete@ @uol.com.br

\begin{abstract}
Resumo
Objetivo: Analisar a toxoplasmose congênita em crianças acompanhadas no Instituto de Perinatologia Social da Maternidade Dona Evangelina Rosa. Metodologia: Tratou-se de um estudo descritivo, quantitativo transversal do tipo pesquisa documental. $\mathrm{O}$ estudo incluiu 85 recém-nascidos que foram expostos a toxoplasmose durante a gestação, nascidos entre Janeiro de 2018 e Dezembro de 2021. A coleta de dados foi realizada por meio das "Fichas de Notificação/Investigação de Toxoplasmose Congênita do Sistema de Agravos de Notificação". Resultados: Verificouse que a maior parte das gestantes estavam na faixa etária de 20 a 29 anos no momento do diagnóstico. Também foi observado a idade gestacional em que a maioria das mães foram diagnosticadas, diagnóstico ocorreu durante o intervalo de 37 semanas a 40 semanas e 6 dias de gestação. Além disso, totalizou-se cerca de $56 \%$ das crianças não apresentaram alterações clínicas. Dentre as crianças sintomáticas, as alterações mais comuns foram retinocoroidite, prematuridade, icterícia, hidrocefalia e ascite. Os resultados mostraram que cerca de 53\% das crianças fizeram uso da combinação de Sulfadiazina +Pirimetamina + Ácido Folínico. Conclusão: Foi possível traçar o perfil epidemiológico de gestantes diagnosticadas com toxoplasmose, como também foi avaliado as manifestações clínicas, o tratamento e a evolução das crianças com toxoplasmose congênita.
\end{abstract}

Palavras-chave: Toxoplasmose congênita; Epidemiologia; Maternidade.

\begin{abstract}
Objective: To analyze congenital toxoplasmosis in children followed at the Social Perinatology Institute of the Dona Evangelina Rosa Maternity Hospital. Methodology: This was a descriptive, quantitative cross-sectional documentary research study. The study included 85 newborns who were exposed to toxoplasmosis during pregnancy, born between January 2018 and December 2021. Data collection was performed using the "Congenital Toxoplasmosis Reporting/Investigation Sheets of the Notifiable Diseases System ". Results: It was found that most pregnant women were aged between 20 and 29 years at the time of diagnosis. The gestational age at which most mothers were diagnosed was also observed, diagnosis occurred during the range of 37 weeks to 40 weeks and 6 days of gestation. In addition, approximately $56 \%$ of the children did not present clinical alterations. Among symptomatic children, the most common alterations were retinochoroiditis, prematurity, jaundice, hydrocephalus and ascites. The results showed that about $53 \%$ of the children used the combination of Sulfadiazine + Pyrimethamine + Folinic Acid. Conclusion: It was possible to trace the epidemiological profile of pregnant women diagnosed with toxoplasmosis, as well as the clinical manifestations, treatment and evolution of children with congenital toxoplasmosis were evaluated.
\end{abstract}

Keywords: Congenital Toxoplasmosis; Epidemiology; Maternity. 


\begin{abstract}
Resumen
Objetivo: Analizar la toxoplasmosis congénita en niños seguidos en el Instituto de Perinatología Social de la Maternidad Doña Evangelina Rosa. Metodología: Se trata de un estudio de investigación documental, descriptivo, cuantitativo y transversal. El estudio incluyó a 85 recién nacidos expuestos a toxoplasmosis durante el embarazo, nacidos entre enero de 2018 y diciembre de 2021. La recolección de datos se realizó utilizando las "Fichas de Reporte / Investigación de Toxoplasmosis Congénita del Sistema de Enfermedades Notificables”. Resultados: Se encontró que la mayoría de las mujeres embarazadas tenían entre 20 y 29 años al momento del diagnóstico. También se observó la edad gestacional en la que la mayoría de las madres fueron diagnosticadas, el diagnóstico ocurrió durante el rango de 37 semanas a 40 semanas y 6 días de gestación. Además, aproximadamente el $56 \%$ de los niños no presentaron alteraciones clínicas. Entre los niños sintomáticos, las alteraciones más frecuentes fueron retinocoroiditis, prematuridad, ictericia, hidrocefalia y ascitis. Los resultados mostraron que aproximadamente el $53 \%$ de los niños usaron la combinación de sulfadiazina + pirimetamina + ácido folínico. Conclusión: Se logró rastrear el perfil epidemiológico de las gestantes diagnosticadas de toxoplasmosis, así como se evaluaron las manifestaciones clínicas, el tratamiento y la evolución de los niños con toxoplasmosis congénita.

Palabras clave: Toxoplasmosis congénita; Epidemiología; Maternidad.
\end{abstract}

\title{
1. Introdução
}

A toxoplasmose congênita é uma doença infecciosa parasitária causada pelo Toxoplasma gondii, parasita intracelular obrigatório, cujos hospedeiros definitivos são os felídeos e os hospedeiros intermediários são o homem, outros mamíferos e as aves (Sousa et al., 2015).

Nos humanos, uma das possiblidades de transmissão é a materno-fetal onde o parasita, adquirido por meio da primoinfecção da mãe, durante a gestação ou próximo à concepção, por reativação de infecção prévia em mães imunodeprimidas, ou ainda por reinfecção de uma gestante com uma nova cepa devido a ingestão de alimentos contaminados, consegue ultrapassar a barreira placentária infectando o concepto (Ducournau et al., 2020).

Ainda convém lembrar que a gravidade das manifestações clínicas varia de acordo com a idade gestacional, ou seja, quanto menor a idade gestacional, maior a probabilidade de morte do recém-nascido e manifestações clínicas severas antes ou depois do nascimento (Sousa et al., 2015). As manifestações clínicas da toxoplasmose congênita incluem sequelas oculares e neurológicas, atraso no desenvolvimento, anormalidades motoras, convulsões e hidrocefalia. (Hutson et al., 2015) Outrossim, calcificações cerebrais, esplenomegalias, perda auditiva, cegueira e morte (Prusa et al., 2017).

Frente ao exposto, faz-se necessário conhecer o perfil clínico e epidemiológico das crianças diagnosticadas com toxoplasmose congênita, evidenciando época de diagnóstico, sintomatologia, tratamento e evolução das mesmas, com o intuito de poder embasar estratégias de diagnóstico precoce, tratamento efetivo, minimizando assim as possíveis sequelas (Zahir et al., 2015).

Ademais, enfatizar a importância da realização do pré-natal, pois a maioria das gestantes infectadas são assintomáticas e aproximadamente $70 \%$ dos lactentes com essa infecção não apresentam sinais clínicos evidentes ao nascimento e $10 \%$ das crianças acometidas apresentam manifestações graves nos primeiros dias de vida. É importante salientar que, se não tratadas corretamente, há desenvolvimento de sequelas na infância ou vida adulta (Maldonado et al., 2017).

O objetivo do trabalho é analisar a toxoplasmose congênita em crianças acompanhadas no Instituto de Perinatologia Social da Maternidade Dona Evangelina Rosa. Identificando o perfil das mães de crianças com toxoplasmose congênita quanto à faixa etária, à idade gestacional que foram diagnosticadas e à procedência. Descrevendo as manifestações clínicas, o tratamento e a evolução das crianças com toxoplasmose congênita.

\section{Metodologia}

Tratou-se de um estudo descritivo, quantitativo transversal do tipo pesquisa documental que foi realizado na Maternidade Dona Evangelina Rosa (MDER), localizada no município de Teresina-Piauí, que é a referência em atendimento 
de gestantes e neonatos de alto risco de todo o Estado do Piauí e também de estados vizinhos. O estudo incluiu 85 recémnascidos (RNs)que foram expostos a toxoplasmose durante a gestação, nascidos entre Janeiro de 2018 e Dezembro de 2021. Foram excluídos RNs notificados com sífilis congênita na MDER, cuja ficha de notificação não foi localizada.

A coleta de dados foi realizada pelas pesquisadoras por meio das "Fichas de Notificação/Investigação de Toxoplasmose Congênita do Sistema de Agravos de Notificação", preenchidas pela equipe responsável na MDER, a partir de casos notificados. Ainda, foi utilizado um formulário para guiar a coleta dos dados, a partir da referida ficha de notificação. Após a fase de coleta, os dados foram compilados em planilhas do Microsoft Excel®2016, e posteriormente, realizadas análises descritivas das variáveis estudadas.

O estudo foi iniciado após autorização da Instituição Co-participante, submissão do projeto à Plataforma Brasil e posterior análise e aprovação pelo Comitê de Ética em Pesquisa do Centro Universitário Uninovafapi, com número do CAE: 44752721.8.0000.5210 e de acordo com a Resolução 466/2012 do Conselho Nacional de Saúde, que rege as teses operacionais e éticas dos trabalhos científicos que envolvem seres humanos.

A pesquisa apresentou riscos mínimos, visto que não houve contato direto com os pacientes estudados e que os próprios autores foram os responsáveis pela coleta de dados conforme o formulário de coleta. Além disso, o risco de invasão de privacidade foi amenizado com a não identificação das fichas durante o manuseio das mesmas.

Não houve benefícios diretos para os participantes da pesquisa. Dentre os benefícios indiretos foi possível a análise de como estão sendo conduzidos os atendimentos de crianças expostas a TC, de acordo com a ficha de notificação preconizada pelo Ministério da Saúde.

\section{Resultados}

Participaram do estudo 85 crianças diagnosticadas com toxoplasmose congênita que fizeram acompanhamento no Instituto de Perinatologia Social da Maternidade Dona Evangelina Rosa durante o período de 2018 a 2020, cujos responsáveis estavam de acordo com os termos da pesquisa, compreendendo e concordando com o Termo de Consentimento Livre Esclarecido, autorizando a participação no estudo.

Os resultados buscaram traçar o perfil das mães de crianças com toxoplasmose congênita quanto à faixa etária, à idade gestacional que foram diagnosticadas e à procedência. A respeito da faixa etária materna no momento do diagnóstico, o grupo majoritário se apresenta na faixa de 20 a 29 anos totalizando 45 mulheres, durantes o período do estudo. Destacando ao ano 2020, onde contabilizou 26 mães dentro desta faixa etária (Figura 1). 
Figura 1: Faixa etária materna no momento do diagnóstico.

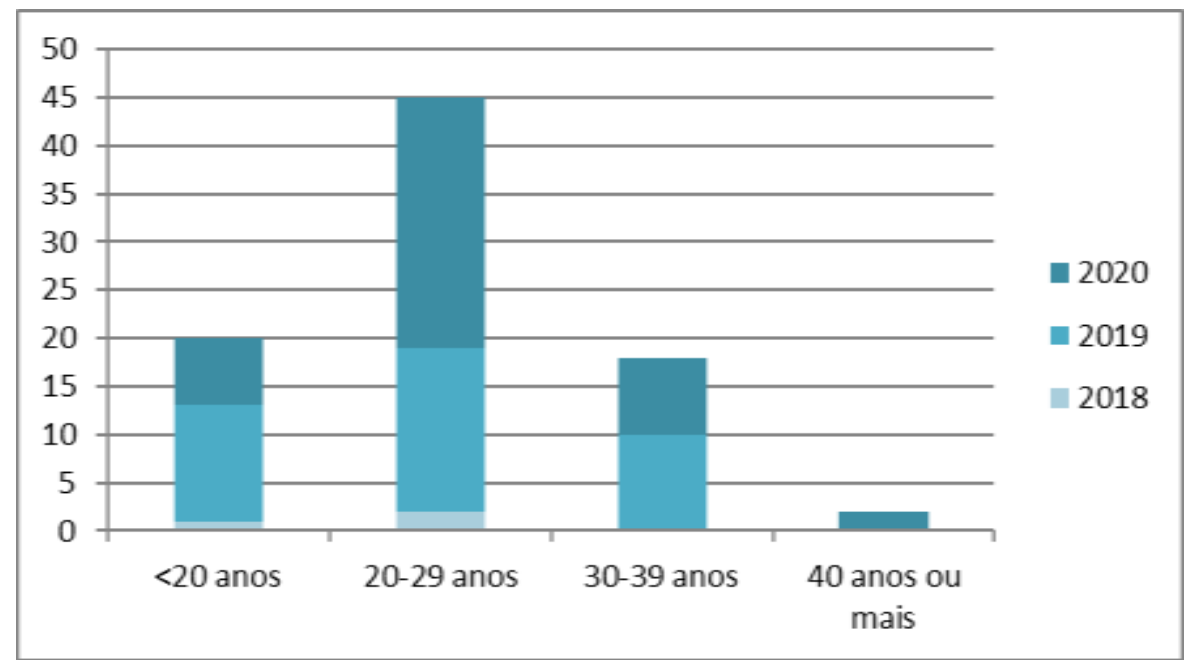

Fonte: Autores.

O segundo ponto abordado foi a idade gestacional que as mães foram diagnosticadas, o que mostra a Figura 2 . A grande maioria teve o diagnóstico de toxoplasmose no intervalo de 37 semanas a 40 semanas e 6 dias de gestação, totalizando 38 participantes. Um número expressivo de participantes, 21 mães não constava a idade gestacional do diagnóstico (Figura 2).

Em relação procedência das mães, foram contabilizadas 63 participantes (74\%) residiam na capital do estado, 19 participantes $(22 \%)$ residiam no interior do estado e apenas $3(4 \%)$ eram oriundas de outro estado (Figura 3). Além disso, outros dados foram importantes na identificação do perfil das mães diagnosticadas com toxoplasmose, como o número de consultas durante o pré-natal, onde a grande maioria, 56 participantes $(65,88 \%)$, realizou 6 ou mais consultas.

Figura 2: Idade gestacional do diagnóstico.

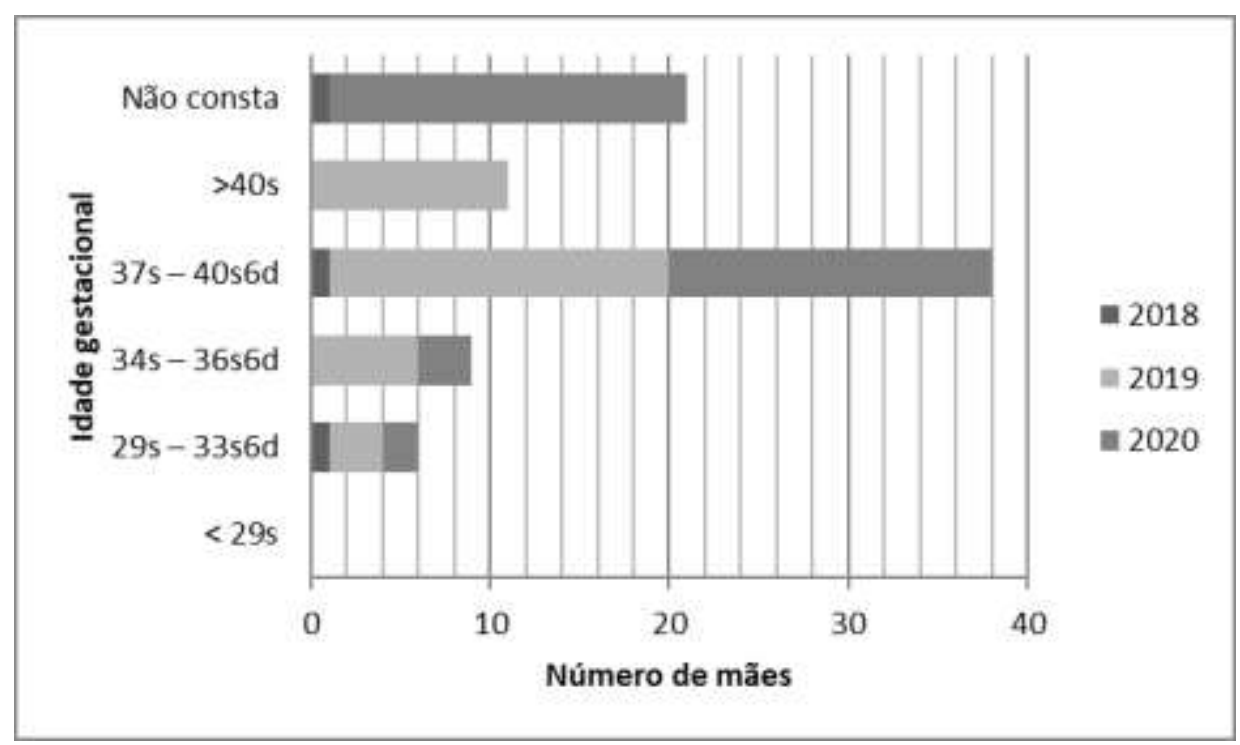

Fonte: Autores. 
Figura 3: Procedências das mães.

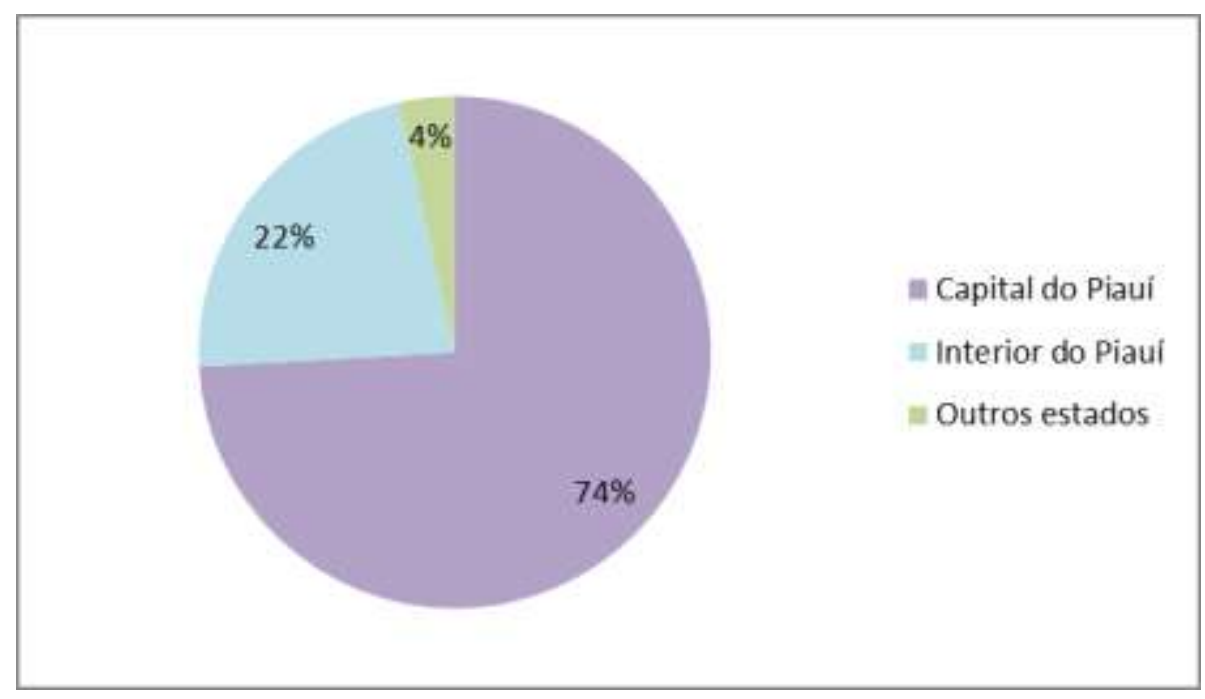

Fonte: Autores.

Outras características maternas apontadas pelo estudo foram os sintomas na gestação e os exames realizados no diagnóstico. A grande maioria das participantes se apresentaram assintomática, 82 mães (96,5\%), apenas 3 mães apresentaram febre e cefaleia. O exame utilizado no diagnóstico foi a sorologia para IgM e IgG, onde 52 participantes (61,2\%) apresentaram IgG e IgM reagentes, 10 participantes $(11,8 \%)$ apresentaram IgM reagente e 15 participantes $(17,64 \%)$ apresentaram $\operatorname{IgG}$ reagente. Sendo a grande maioria, cerca de $91 \%$ das participantes do estudo foram positivo para alguma imunoglobulina (Figura 4).

Figura 4: Sorologia materna para detecção de IgM e IgG.

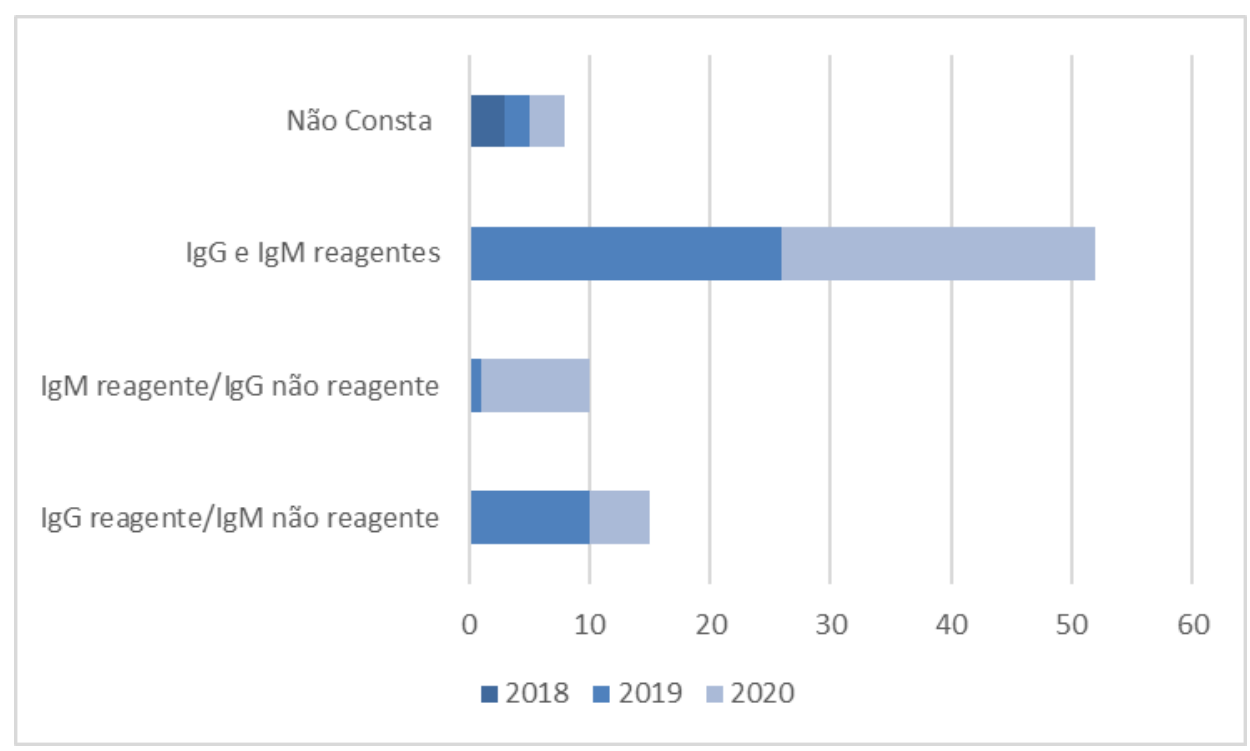

Fonte: Autores.

A pesquisa também abordou as manifestações clínicas, o tratamento e a evolução das crianças com toxoplasmose congênita atendidas no Instituto de Perinatologia Social da Maternidade Dona Evangelina Rosa. Dentre as crianças que participaram do estudo, a maioria foi identificada como do sexo feminino, 52 crianças (61\%), 31 foram identificadas como do sexo masculino ( $37 \%)$ e dois bebês $(2 \%)$ não foram identificados. 
Dentro das manifestações clínicas, a figura 5 traz as alterações presentes nas crianças ao exame físico na sala de parto, onde os resultados mostram que o grupo majoritário das crianças não possuía nenhuma alteração, cerca de 56\%. As alterações mais comuns foram retinocoroidite (4\%), prematuridade (2\%), icterícia (2\%), hidrocefalia (1\%) e ascite (1\%). Ressaltando que $34 \%$ das crianças participantes não constavam essa informação. Também foi avaliado se o fundo de olho dos bebês se encontrava alterado, apenas 5 crianças apresentaram o fundo de olho alterado, 37 crianças com fundo de olho normal e a grande maioria, 43 crianças não registram esse dado.

Figura 5: Alterações presentes nas crianças ao exame físico na sala de parto.

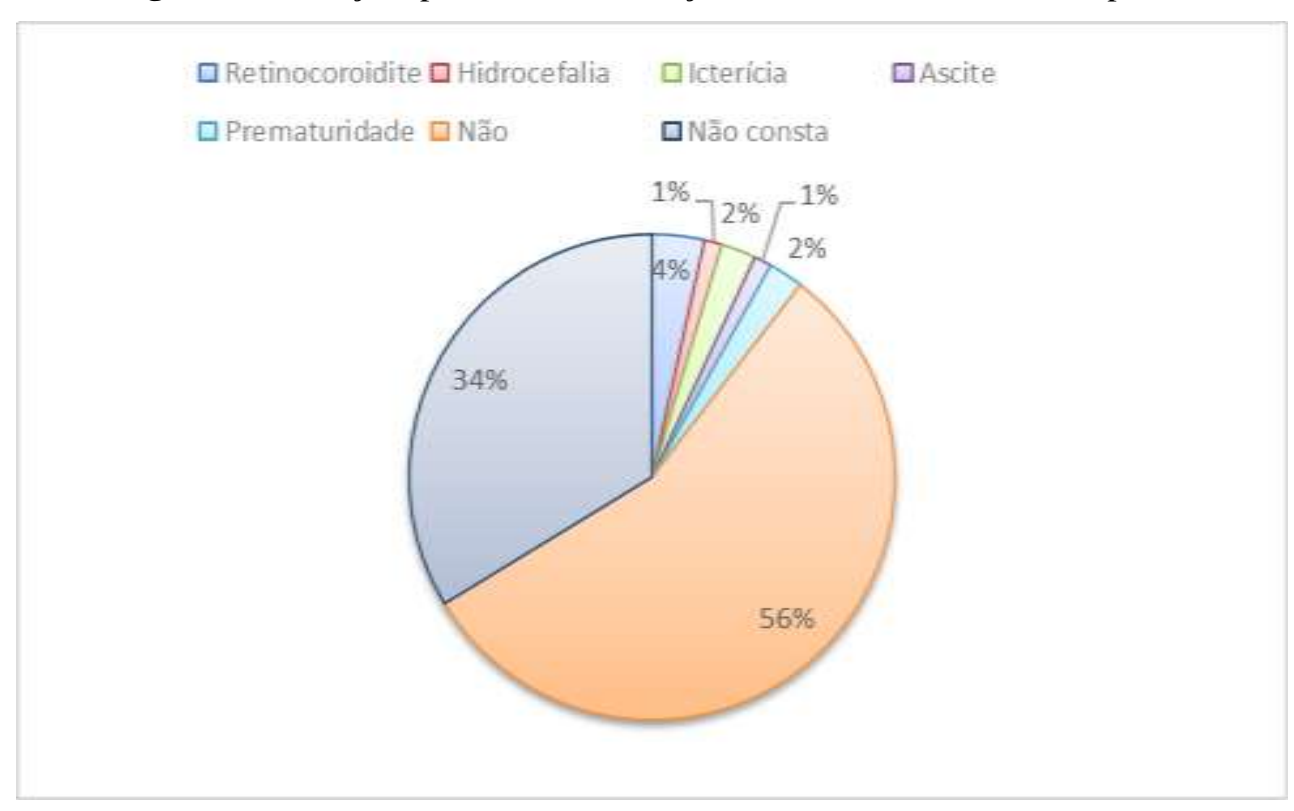

Fonte: Autores.

Outro aspecto importante avaliado dentro da pesquisa foi o exame realizado para o diagnóstico de toxoplasmose, a metodologia utilizada foi a sorologia para detecção de IgM e IgG. Os resultados mostraram que 47 crianças apresentam sorologia reagente para IgG e 10 crianças com sorologia reagente para IgM, ou seja, cerca de 67\% dos participantes apresentaram o diagnóstico positivo para alguma das imunoglobulinas (Figura 6). 
Figura 6: Sorologia das crianças para detecção de $\operatorname{IgM}$ e IgG.

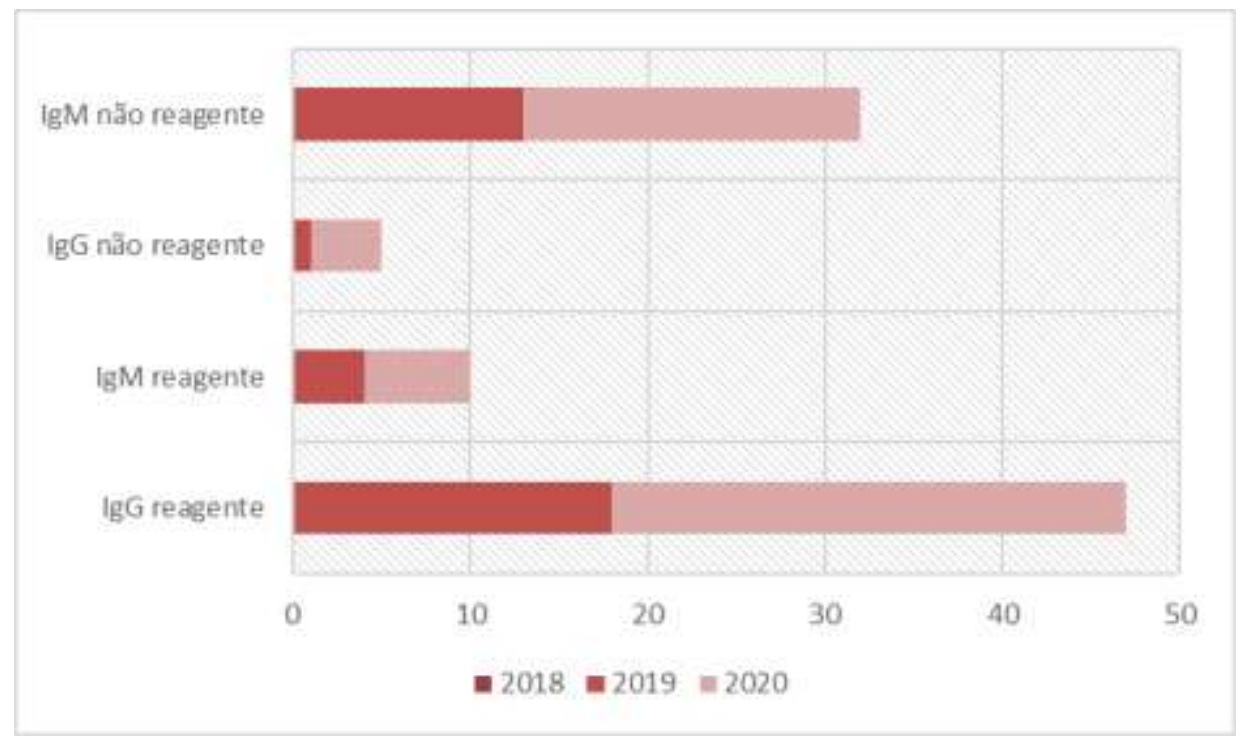

Fonte: Autores.

Por fim, o estudo avaliou o tratamento utilizado e a evolução das crianças com toxoplasmose congênita. De acordo com a Figura 7, a maioria dos participantes, 45 crianças $(52,92 \%)$ fizeram uso da combinação de Sulfadiazina +Pirimetamina + Ácido Folínico. Por outro lado, um número expressivo de participantes, 37 crianças (43,52\%) não fizeram uso de nenhuma medicação. 3 crianças $(3,5 \%)$ fizeram uso incompleto do tratamento. O ano de 2020 apresentou o maior número de crianças sem tratamento, totalizando 33 crianças.

Figura 7: Tratamento utilizado pelas crianças com toxoplasmose congênita.

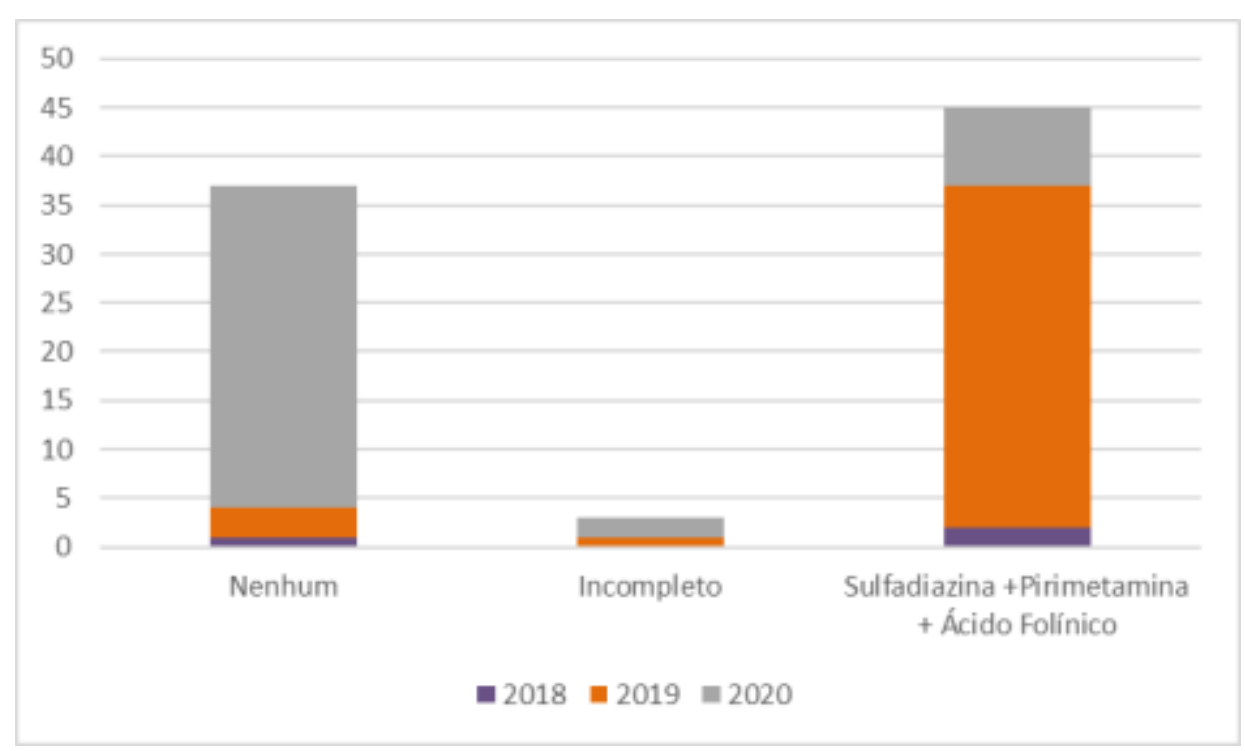

Fonte: Autores.

\section{Discussão}

A toxoplasmose é uma zoonose que possui uma distribuição universal, causada pelo Toxoplasma gondii, um parasita intracelular. Fatores como alimentação, higiene, população, conato com gatos e climas quentes influenciam no índice de 
infeç̧ão da doença. A prevalência da infecção varia de região para região sendo mais comum em países tropicais. A toxoplasmose é uma infecção parasitária que acomete frequentemente o ser humano podendo o afetar negativamente e o seu feto em desenvolvimento (Hampton, 2015; El Bissati et al., 2018; Peyron et al., 2019).

O risco de transmissão meterno-fetal varia de $10 \%$ a $80 \%$, tal variação está relacionada a idade gestacional do feto, resposta imune da mãe, virulência da cepa do parasita, a carga parasitária, permeabilidade da placenta, além da existência de um tratamento pré-natal. Dessa forma, a infecção parasitaria em grávidas tem resultado prejudicial maior, ou seja, o grau de comprometimento do feto é mais grave quando a transmissão ocorre no início da gestação (Fonseca et al., 2016; Ducournau et al., 2020).

De acordo com os resultados da pesquisa, a idade gestacional em que a maior parte das mães foram diagnosticadas com toxoplasmose foi durante o intervalo de 37 semanas a 40 semanas e 6 dias de gestação, cerca de $44 \%$ das participantes. Isso é explicado devido ao fato que o terceiro trimestre de gestação é período gestacional considerado com maior risco de transmissão vertical (Maldonado et al., 2017; Olariu et al., 2019; Conceição, 2020).

Diferentemente, do que foi observado no estudo de Conceição (2020), que o período gestacional de maior ocorrência das notificações é o segundo trimestre de gestação. Embora o diagnóstico de infecção pelo T. gondii seja de suma importância, percebemos que o diagnóstico se deu de forma tardia, já no final de gestação, retardando o início da terapia protocolar para que se minimize as complicações clínicas no feto (Walcher \& 2017; Takahashi et al., 2019).

O estudo de Moura e colaboradores (2018), semelhante ao presente estudo, buscou traçar o perfil epidemiológico de gestantes diagnosticadas com toxoplasmose congênita em um município do estado do Alagoas. A respeito da faixa etária no momento do diagnóstico, verificou-se que 30 das 40 gestantes analisadas tinham menos de 25 anos, corroborando com os nossos resultados, o grupo majoritário se apresenta na faixa de 20 a 29 anos. Também foi apontado que o desconhecimento das gestantes sobre a toxoplasmose está relacionado à falta de orientações e informações dos profissionais de saúde durante as consultas de pré-natal.

Dentro do contexto das manifestações clínicas das crianças com toxoplasmose congênita, cerca de $70 \%$ dos recémnascidos são assintomáticos ao nascimento e por volta de $10 \%$ apresenta manifestações sérias nos primeiros dias de vida. A forma congênita da doença tem um desenvolvimento multissistêmico ou com comprometimento do sistema nervoso, podendo estar associado a forma ocular. Existindo a possibilidade de sobreposição das manifestações clínicas somadas a manifestações inespecíficas (Thakkar et al., 2018; Gómez-Toscano et al., 2019).

O estudo de Almeida (2017) buscou identificar as principais manifestações da toxoplasmose em crianças com a infecção parasitária associando ao histórico gestacional e tratamento materno. Os resultados mostraram que apenas 29\% dos recém-nascidos foram assintomáticos, dentre os recém-nascidos com toxoplasmose congênita sintomáticos os achados mais comuns foram hepatomegalia com $35(71,4 \%)$ casos, coriorretinite com $45(65,2 \%)$ casos, icterícia com 21 (42,8\%) casos, prematuridade com $24(34,8 \%)$ casos e microcefalia com $16(32,6 \%)$ casos. Diferentemente do presente estudo, que apresentou um índice mais alto de RNs assintomáticos, cerca de 56\% das crianças não apresentaram alterações clínicas. As alterações mais comuns foram retinocoroidite (4\%), prematuridade (2\%), icterícia (2\%), hidrocefalia (1\%) e ascite (1\%).

Existe uma grande variedade de métodos para o diagnostico da toxoplasmose como histológicos, parasitológicos, isolamento do parasita in vivo e in vitro e testes moleculares, entretanto, a metodologia de escolha é a sorologia utilizando imunoensaios, pois além de determinar a doença consegue definir a fase clínica da mesma. Os exames sorológicos permitem detectar a presença de imunoglobulinas anti-T.gondii (IgG, IgM, IgA) que aparecem após a infecção (Walcher et al., 2017; Goncalves et al., 2019).

No presente trabalho, exame utilizado no diagnóstico foi a sorologia para IgM e IgG, onde 52 participantes $(61,2 \%)$ apresentaram IgG e IgM reagentes, 10 participantes $(11,8 \%)$ apresentaram $\operatorname{IgM}$ reagente e 15 participantes $(17,64 \%)$ 
apresentaram IgG reagente. Sendo a grande maioria, cerca de $91 \%$ das participantes do estudo foram positivo para alguma imunoglobulina.

O estudo de Inagaki e colaboradores (2014) teve como objetivo analisar a distribuição espacial da prevalência de anticorpos antitoxoplasma em gestantes residentes em uma cidade do Nordeste do Brasil utilizando como exame de diagnostico a sorologia. Os resultados mostraram que a soroprevalência encontrada para IgG foi de $68,5 \%$ e IgM de $0,36 \%$.

O último aspecto avaliado foi o tratamento utilizado e a evolução das crianças com toxoplasmose congênita. Segundo o Ministério da Saúde, o protocolo de tratamento deve iniciar com o uso de espiramicina logo após o diagnóstico da infecção materna. Em casos de alteração fetal, deve ser feita a troca por sulfadiazina associada a pirimetamina. Além disso, recomendase adicionar ao esquema o ácido folínico, na intenção de minimizar os efeitos adversos causados pela pirimetamina e caracterizados por supressão da medula óssea e consequenteanemia e neutropenia. Os resultados do estudo mostraram que cerca de 53\% das crianças fizeram uso da combinação de Sulfadiazina +Pirimetamina + Ácido Folínico.

\section{Conclusão}

A toxoplasmose é uma infecção parasitária que acomete frequentemente o ser humano podendo o afetar de forma negativa e o seu feto em desenvolvimento. O estudo apresentou o perfil epidemiológico de gestantes diagnosticadas com toxoplasmose, onde verificou-se que a maior parte das participantes estava na faixa etária de 20 a 29 anos no momento do diagnóstico. Também foi observado a idade gestacional em que a maior parte das mães foram diagnosticadas, ocorrendo durante o intervalo de 37 semanas a 40 semanas e 6 dias de gestação.

Cerca de $56 \%$ das crianças não apresentaram alterações clínicas e as alterações mais comuns foram retinocoroidite, prematuridade, icterícia, hidrocefalia e ascite. Os resultados mostraram que cerca de 53\% das crianças fizeram uso da combinação de Sulfadiazina +Pirimetamina + Ácido Folínico. Dessa forma, Foi possível traçar o perfil epidemiológico de gestantes diagnosticadas com toxoplasmose, como também foi avaliado as manifestações clínicas, o tratamento e a evolução das crianças com toxoplasmose congênita.

\section{Referências}

Almeida, M. I. D. B. (2017). Associação das manifestações da toxoplasmose congênita em recém-nascidos e lactentes com a história gestacional e tratamento materno. Tese (Doutorado).

Conceição, C. D. S. (2020). Perfil sociodemográfico e obstétrico de gestantes notificadas por toxoplasmose. (Manografia).

de Moura, D. S., Oliveira, R. D. C. M., \& Matos-Rocha, T. J. (2018). Toxoplasmose gestacional: perfil epidemiológico e conhecimentos das gestantes atendidas na unidade básica de saúde de um município alagoano/Toxoplasmosis in pregnancy: Epidemiological profile and knowledge of pregnant women assisted in basic units of an Alagoan municipality. Arquivos Médicos dos Hospitais e da Faculdade de Ciências Médicas da Santa Casa de São Paulo, 63(2), 69-76.

Ducournau, C., Moiré, N., Carpentier, R., Cantin, P., Herkt, C., Lantier, I., \& Dimier-Poisson, I. (2020). Effective nanoparticle-based nasal vaccine against latent and congenital toxoplasmosis in sheep. Frontiers in Immunology, 11, 2183.

El Bissati, K., Levigne, P., Lykins, J., Adlaoui, E. B., Barkat, A., Berraho, A., \& McLeod, R. (2018). Global initiative for congenital toxoplasmosis: an observational and international comparative clinical analysis. Emerging microbes \& infections, 7(1), 1-14.

Fonseca, Z. C., Rodrigues, I. M. X., e Melo, N. C., Castro, A. M., \& Avelino, M. M. (2016). Importância do teste de avidez IgG na toxoplasmose congênita. Revista de Patologia Tropical/Journal of Tropical Pathology, 45(1), 42-54.

Gómez-Toscano, V., Linares-López, K. A., Arce-Estrada, G. E., Figueroa-Damián, R., Barrios-Bautista, D. M., Hernández-Luengas, L., \& Correa, D. (2019). Toxoplasmosis congénita en el valle de México. Resultados de una serie de casos. Acta Pediátrica de México, 39(6), $321-333$.

Goncalves, D. D., Silva, B. C., Lopes, L. F., Diegas, P. H., Teixeira, V. S., \& Esteves, A. P. V. (2019). Toxoplasmose congênita: Estratégias de controle durante o pré-natal. Cadernos da Medicina-UNIFESO, 2(1).

Hampton, M. M. (2015). Congenital toxoplasmosis: a review. Neonatal Network, 34(5), 274-278.

Hutson, S. L., Wheeler, K. M., McLone, D., Frim, D., Penn, R., Swisher, C. N., \& McLeod, R. (2015). Patterns of hydrocephalus caused by congenital Toxoplasma gondii infection associate with parasite genetics. Clinical Infectious Diseases, 61(12), 1831-1834. 
Research, Society and Development, v. 10, n. 17, e81101724524, 2021

(CC BY 4.0) | ISSN 2525-3409 | DOI: http://dx.doi.org/10.33448/rsd-v10i17.24524

Inagaki, A. D. D. M., Cardoso, N. P., Lopes, R. J. P. L., Alves, J. A. B., Mesquita, J. R. F., Araújo, K. C. G. M. D., \& Katagiri, S. (2014). Análise espacial da prevalência de toxoplasmose em gestantes de Aracaju, Sergipe, Brasil. Revista Brasileira de Ginecologia e Obstetrícia, 36, 535-540.

Maldonado, Y. A., Read, J. S., Byington, C. L., Barnett, E. D., Davies, H., Edwards, K. M., \& COMMITTEE ON INFECTIOUS DISEASES. (2017). Diagnosis, treatment, and prevention of congenital toxoplasmosis in the United States. Pediatrics, 139(2).

Olariu, T. R., Press, C., Talucod, J., Olson, K., \& Montoya, J. G. (2019). Congenital toxoplasmosis in the United States: clinical and serologic findings in infants born to mothers treated during pregnancy. Parasite, 26.

Peyron, F., L'ollivier, C., Mandelbrot, L., Wallon, M., Piarroux, R., Kieffer, F., \& Garcia-Meric, P. (2019). Maternal and congenital toxoplasmosis: diagnosis and treatment recommendations of a French multidisciplinary working group. Pathogens, 8(1), 24.

Prusa, A. R., Kasper, D. C., Sawers, L., Walter, E., Hayde, M., \& Stillwaggon, E. (2017). Congenital toxoplasmosis in Austria: Prenatal screening for prevention is cost-saving. PLoS neglected tropical diseases, 11(7), e0005648.

Sociedade Brasileira de Pediatria. (2020). Toxoplasmose Congênita.

Souza, C. Z., Marchioro, A. A., Rafael, K., Araújo, S. M., \& Falavigna-Guilherme, A. L. (2015). Aborto espontâneo e toxoplasmose ocular em um casal infectado com Toxoplasma gondii. Scientia Medica, 25(3), ID21119-ID21119.

Takahashi, A. F. S., Bioni, H. O., de Souza, J. M., Takizawa, M. D. G. M. H., \& Paiva, J. E. (2019). Toxoplasmose congênita na cidade de Cascavel/PR no período de 2002-2016. Revista Thêma et Scientia, 9(1), 260-267.

Thakkar, P. A., Shah, A., Javadekar, B. B., \& Modasia, S. (2018). Congenital Toxoplasmosis presenting as multiple ring enhancing lesions in brain on neuroimaging. International Journal of Contemporary Pediatrics, 5(5), 2020.

Walcher, D. L., Comparsi, B., \& Pedroso, D. (2017). Toxoplasmose gestacional: uma revisão. Brazilian Journal of Clinical Analyses, 49(4), 323-7.

Zahir, F., Abdellaoui, M., Younes, S., Benatiya, I. A., \& Tahri, H. (2015). Severe ocular sequelae of congenital toxoplasmosis: huge macular scar. The Pan African Medical Journal, 20. 